\title{
Erratum
}

\section{Direct Conversion of Olefins into $\alpha$-Bromo Ketones Using $O$-Iodoxybenzoic Acid and Tetraethylammonium Bromide}

Swapnil S. Deshmukh, Kiran H. Chaudhari, Krishnacharya G. Akamanchi* Synlett 2011, 81.

In the advance online publication (e-First) version of this article the products of entry 1 and 2 in Table 1 were interchanged. This has been corrected for both the current online version and the print publication. We apologize for this error. 